\title{
General Measures of Infection Control
}

\author{
Sheila Nainan Myatra and Jacob George Pulinilkunnathil
}

A 72-year-old male patient with non-Hodgkin's lymphoma was neutropenic after chemotherapy and presented to the ICU with breathlessness and hypotension. He was intubated and kept on a ventilator and received broad-spectrum antibiotics. He had a peripheral, central, and arterial line in place. A Foley's catheter and a nasogastric tube were also placed.

Health-care-associated infections are a common cause of increased morbidity, mortality, and cost of care in ICUs. A systematic and multidisciplinary approach to infection control practice goes a long way in minimizing this problem. Infectious patients needs to be isolated to prevent spread of infection to other patients and to healthcare staff. Apart from appropriate isolation precautions, infection control and judicious antibiotic use are the mainstay of management of these patients.

\section{Step 1: Assess the Need for Isolation}

- Screen all ICU patients for the following:

- Neutropenia and immunological disorder.

- Diarrhea.

- Skin rashes.

- Known communicable disease.

- Known carriers of an epidemic strain of bacterium.

\section{Step 2: Identify the Type of Isolation Needed}

- There are two types of isolation in the ICU:

\footnotetext{
S. N. Myatra $(\bowtie)$ · J. G. Pulinilkunnathil

Department of Anaesthesia, Critical Care and Pain, Tata Memorial Hospital, Mumbai, India

R. Chawla, S. Todi (eds.), ICU Protocols, https://doi.org/10.1007/978-981-15-0898-1_50
} 
- Protective isolation for neutropenic or other immunocompromised patients to reduce the chances of acquiring opportunistic infections.

- Source isolation of colonized or infected patients to minimize potential transmission to other patients or staff.

- Isolation rooms should have tight-fitting doors, glass partitions for observation, and both negative-pressure (for source isolation) and positive-pressure (for protective isolation) ventilations.

\section{Step 3: Identify the Patient at Risk of Nosocomial Infections}

- There are patient-, therapy-, and environment-related risk factors for the development of nosocomial infection:

- Age more than 70 years.

- Shock.

- Major trauma.

- Acute renal failure.

- Coma.

- Prior antibiotics.

- Mechanical ventilation.

- Drugs affecting the immune system (steroids, chemotherapy).

- Indwelling catheters.

- Prolonged ICU stay (>3 days).

\section{Step 4: Observe Hand Hygiene}

- Hands are the most common vehicle for transmission of organisms, and "hand hygiene" is the single most effective means of preventing the horizontal transmission of infections among hospital patients and health-care personnel.

- When and why_follow WHO's five moments for hand hygiene (Fig. 50.1):

1. Before touching a patient - to protect the patient from harmful germs carried on your hands

2. Before aseptic procedures - to protect the patient against harmful germs, including the patient's own germs

3. After body fluid exposure/risk - to protect yourself and the health-care environment from the harmful patient's germs

4. After touching the patient - to protect yourself and the health-care environment from the harmful patient's germs

5. After touching the patient's surrounding - to protect yourself and the health-care environment from the harmful patient's germs

(Remember, there are two moments before and three moments after touching the patient) 


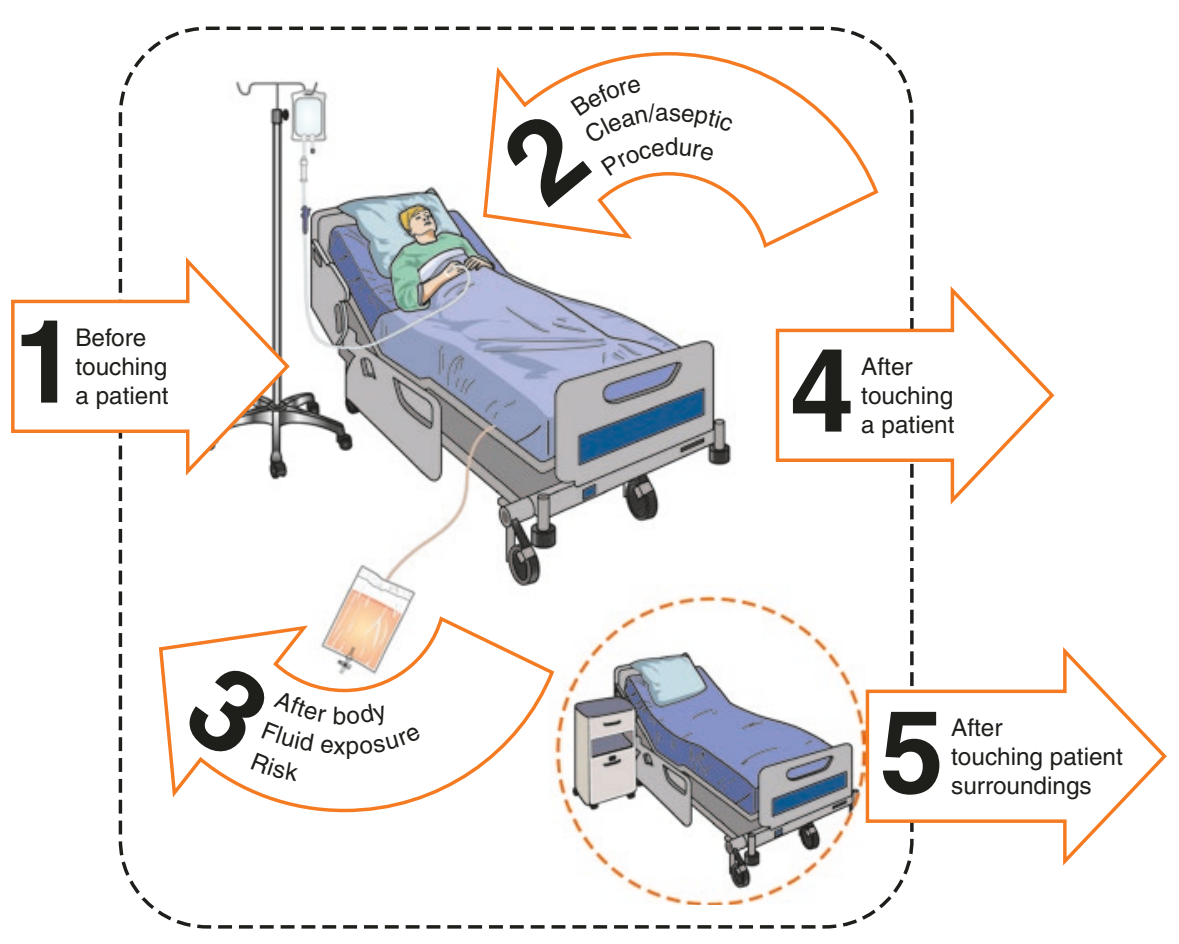

Fig. 50.1 WHO's five moments for hand hygiene (Adapted from WHO)

- How

- Wash hands with soap and water when they are soiled or visibly dirty with blood or other body fluids. Wet your hands, apply soap and then scrub them vigorously for at least $15 \mathrm{~s}$. Cover all surfaces of the hands and fingers, wash with water, and then dry thoroughly using a disposable towel (Fig. 48.1).

- Use an alcohol-based hand rub (e.g., $0.5 \%$ chlorhexidine with $70 \%$ w/v ethanol) if hands are not visibly dirty. A combination of chlorhexidine and alcohol is ideal as they cover Gram-positive and Gram-negative organisms, viruses, mycobacteria, and fungi. Chlorhexidine also has residual activity.

- During surgical hand preparation, all hand jewelries (e.g., rings, watches, bracelets) must be removed.

- Finger nails should be trimmed with no nail polish or artificial nails.

- Avoid wearing long sleeves, ties should be tucked in, house coats are discouraged, and wearing scrubs is encouraged.

\section{Step 5: Follow Standard Precautions}

- Standard precautions include prudent preventive measures to be used at all times, regardless of a patient's infection status. Use personal protective equipment (PPE) like gloves, gown, mask, shoe and head covering etc. 
- Gloves

- Sterile gloves should be worn after hand hygiene procedure while touching mucous membrane and nonintact skin and performing sterile procedures (e.g., arterial, central line, and Foley catheter insertion).

- Clean, nonsterile gloves are safe for touching blood, other body fluids, contaminated items, and any other potentially infectious materials.

- Change gloves between tasks and procedures in the same patient especially when moving from a contaminated body area to a clean body area.

- Never wear the same pair of gloves for the care of more than one patient.

- Remove gloves after caring for a patient.

- Practice hand hygiene whenever gloves are removed.

- Gown

- Wear a gown to prevent soiling of clothing and skin during procedures that are likely to generate splashes of blood, body fluids, secretions, or excretions.

- The sterile gown is required only for aseptic procedures, and for the rest, a clean, nonsterile gown is sufficient.

- Remove the soiled gown as soon as possible, with care to avoid contamination.

- Mask, eye protection/face shield

- Wear a mask and adequate eye protection (eyeglasses are not enough) or a face shield to protect mucous membranes of the eyes, nose, and mouth during procedures and patient-care activities that are likely to generate splashes/ sprays of blood, body fluids, etc.

- Patients, relatives, and health-care workers presenting with respiratory symptoms should also use masks (e.g., cough).

- Shoe and head coverings

- They are not required for routine care.

- Patient-care equipment

- Used patient-care equipment soiled with blood, body fluids, secretions, or excretions should be handled carefully to prevent skin and mucous membrane exposures, contamination of clothing, and transfer of microorganisms to health-care workers, other patients, or the environment.

- Ensure that reusable equipment is not used for the care of another patient until it has been cleaned and sterilized appropriately.

- Ensure that single-use items and sharps are discarded properly.

\section{Step 6: Follow Transmission-Based Precautions}

In addition to standard precautions, the following should be observed in those patients known or suspected to have airborne, contact, or droplet infections. See Table 50.1 for details. 


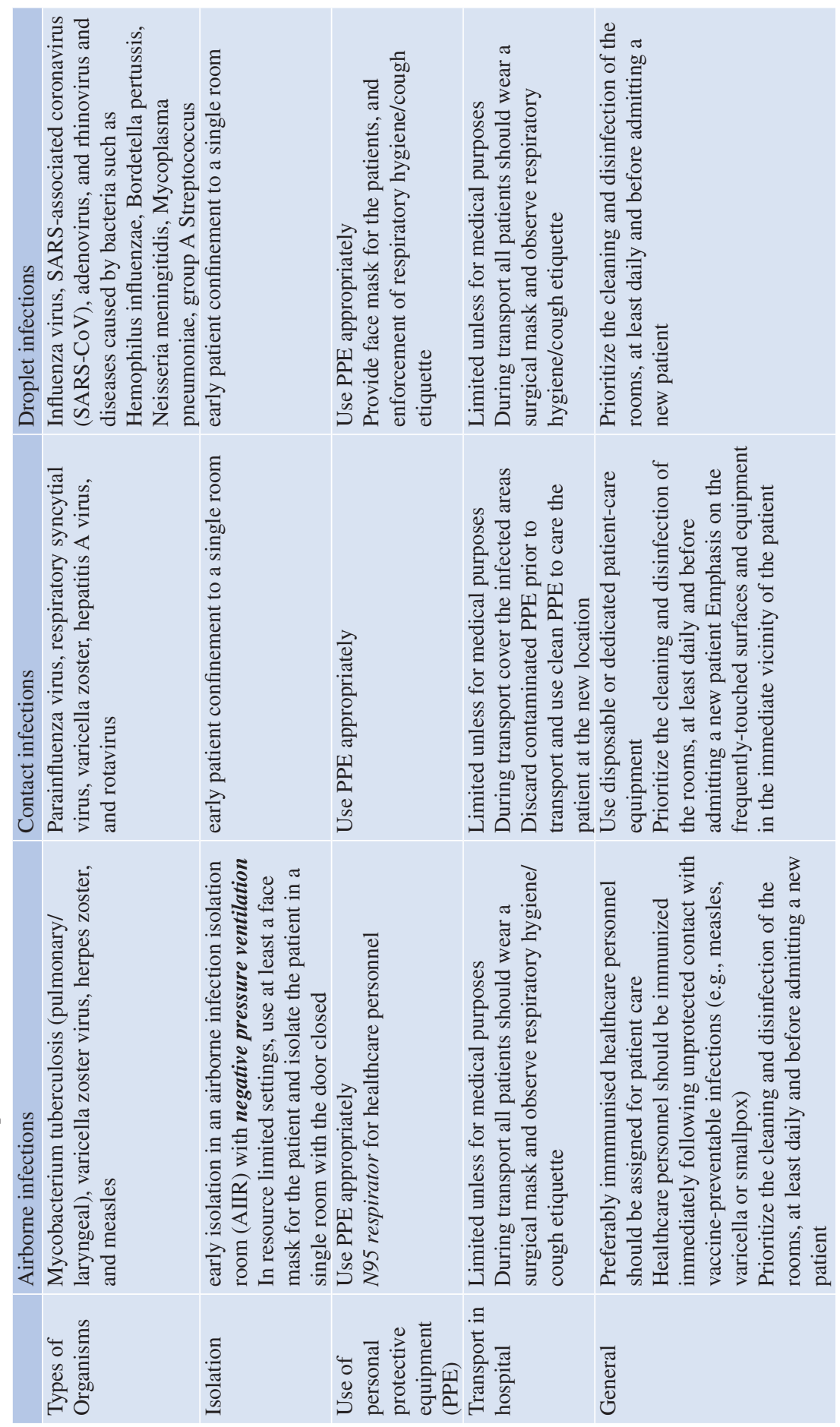




\section{Airborne Infections}

Pathogenic microorganisms may be suspended in the air as small particles, aerosols, or dust and remain infective over time and distance.

\section{Contact Infections}

Some infections spread by direct or indirect contact with an infected person, and also from the surfaces or patient-care items in the room.

\section{Droplet Infections}

Microorganisms can also be transmitted by large droplets $>5 \mu \mathrm{m}$ in size that are generated during coughing, sneezing or talking.

\section{Step 7: Use specific Strategies Focused on Prevention of Specific Nosocomial Infections}

In addition to the standard and transmission-based precautions, there are several strategies focused on prevention of specific nosocomial infections in critically ill patients. Of these, ventilator-associated pneumonia (VAP), catheter-related bloodstream infection (CRBSI), and urinary tract infection (UTI) are the most important.

\section{General Strategies to Reduce VAP (SHEA/IDSA Practice Recommendation 2014)}

- Avoid intubation whenever possible.

- Consider noninvasive ventilation whenever possible.

- Prefer oral intubations to nasal unless contraindicated.

- Minimum sedation for patients, daily interruption of sedation, daily extubation trials to be practiced together.

- Keep head elevated at $30-45^{\circ}$ in the semi-recumbent body position.

- Avoid reintubation whenever possible.

- Routine change of ventilator circuits is not required unless visibly soiled or malfunctioning

- Monitor endotracheal tube cuff pressure (keep it between 20-30 cmH2O) to avoid air leaks around the cuff, which can allow entry of bacterial pathogens into the lower respiratory tract, while permitting capillary perfusion.

- Prefer endotracheal tubes with a subglottic suction port to prevent pooling of secretions around the cuff leading to microaspiration for patients expected to require greater than 48 or 72 hours of mechanical ventilation 
Table 50.2 VAP bundle proposed by the Institute of Healthcare Improvement (2012)

- Head end elevation

- Daily sedation interruption

- Deep vein thrombosis

- Stress ulcer prophylaxis

- Daily use of oral chlorhexidine for oral hygiene

Facilitate early mobility

- Deep vein thrombosis propylaxis

- Stress ulcer prophylaxis

- The initial VAP bundle was proposed by the Institute of Healthcare Improvement in 2012. It includes 5 commponents (Table 50.2) The last three elements have come under scrutiny and many interntional bodies do not include them in the VAP bundle, and many other societies have come up with their VAP bundles, as per the local prevailing policies and available scientific evidences.

\section{General Strategies to Reduce Catheter-Related Infections (CDC 2011-Updated 2017)}

Intravascular catheter related infections can be prevented by maintaining good aseptic practices drug insertion and during catheter handling. There are certain good practices to prevent CRBSI that include.

- Use of Chlorhexidine for daily bath in patients aged more than 2 months

- Avoidance of femoral site for planned CVC insertion in adults and obese patients. The preferred site in adult patients is the subclavian with due consideration regarding the risk for mechanical complications and subclavian vein stenosis. Femoral lines are preferably avoided due to higher rate of infection and thrombosis risks. In case a femoral catheter was inserted in emergency, it is preferable to change it to to an upper extremity site as soon as possible

- The central line site should be dressed with a sterile gauze or sterile, transparent, semipermeable dressing and should be changed with clean or sterile gloves only if it becomes damp, loose, or visibly soiled

- The catheter insertion site should be inspected daily for any signs of infection

- The need for the intravascular catheter should be assessed daily and removed when not required.

- All administration sets should be changed appropriately e.g - every day in patients receiving blood, blood products, or fat emulsions. Every 6 or $12 \mathrm{~h}$ for propofol, and no less than 96-h intervals and at least every 7 days for routine IV sets.

- The needleless connectors should be changed every $72 \mathrm{~h}$ and all disposable or reusable transducers should be changed at 96-h intervals. 
Table 50.3 The central line bundle by Institute of Healthcare Improvement

- Hand hygiene

- Maximal barrier precautions

- Chlorhexidine skin antisepsis

- Optimal catheter site selection, with avoidance of using the femoral vein for central venous access in adult patients

- Daily review of line necessity, with prompt removal of unnecessary lines

- Other practices include adequate staffing with maintained nurse-to-patient ratio, use of antimicrobial ointments at hemodialysis catheter-insertion site and to have a continuous surveillance for CLABSI in both ICU and non-ICU settings.

In spite of adequate precautions, if the CLABSI rate is still high, then additional measures such as

- Use of antiseptic- or antimicrobial-impregnated CVCs in adult patients

- Use chlorhexidine-containing dressings in patients over 2 months of age

- Use of an antiseptic-containing hub/connector cap/port protector to cover connectors may be tried.

The use of antimicrobial locks CVCs are presently recommended only for longterm hemodialysis catheters, in patients with limited venous access and a history of recurrent CLABSI and in patients who are at increased risk of severe complications from CLABSI (e.g patients with prosthetic valves or aortic graft).

The central line bundle by Institute of Healthcare Improvement is in Table 50.3.

\section{General Strategies to Reduce UTI (CDC 2009)}

- Insert catheters only for appropriate indications.

- Follow aseptic insertion of the urinary catheter.

- Maintain a closed drainage system.

- Maintain unobstructed urine flow. At all times, the urinary catheter should be placed and taped above the thigh and the urinary bag should hang below the level of the bladder.

- The urinary bag should never have floor contact.

- Changing indwelling catheters or drainage bags at fixed intervals is not recommended. Change only if there are clinical indications such as infection or obstruction or when the closed system is compromised.

- Remove the catheter when it is no longer needed.

The Catheter Associated Urinary Tract Infection (CAUTI) Bundle from the International Federation of Infection Control (2016) is given in Table 50.4. 
Table 50.4 The CAUTI BUNDLE from the International Federation of Infection Control (2016)

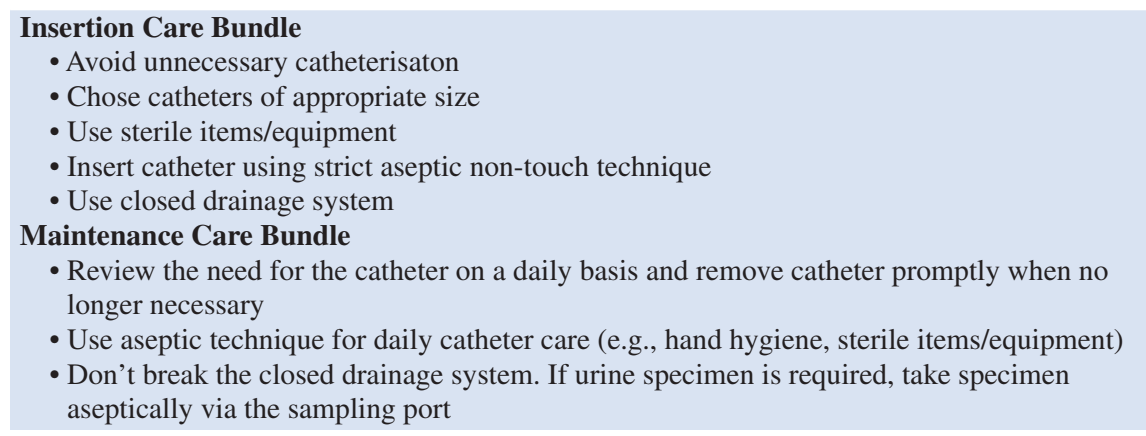

\section{Step 8: Consider Environmental Factors}

- Cleaning and disinfection

- Maintain a high-quality cleaning and disinfection of all patient-care areas, especially bedrails, bedside tables, doorknobs, and equipment.

- EPA-registered disinfectants or detergents that best meet the overall needs of the ICU should be used for routine cleaning and disinfection.

- Schedule of cleaning should be as atlas twice weekly for surface cleaning, twice of thrice a day floor cleaning, and terminal cleaning (patient bed area) after discharge or death.

- Architecture and layout, especially while designing a new ICU

- The unit may be situated close to the operating theater or emergency department for easy accessibility but should be away from the main ward areas.

- Central air-conditioning systems are designed in such a way that recirculated air must pass through appropriate filters.

- It is recommended that all air should be filtered to $99 \%$ efficiency down to $5 \mu \mathrm{m}$.

- Suitable and safe air quality must be maintained at all times. Air movement should always be from clean to dirty areas.

- It is recommended to have a minimum of six total air changes per room per hour, with two air changes per hour composed of outside air. A relative humidity of $30-60 \%$ and temperature of $21-24{ }^{\circ} \mathrm{C}$

- Isolation facility should be with both negative- and positive-pressure ventilations.

- Clearly demarcated routes of traffic flow through the ICU are required.

- Adequate space around beds is ideally 2.5-3 m.

- Electricity, air, vacuum outlets/connections should not hamper access around the bed.

- Adequate number of washbasins should be installed.

- Alcohol gel dispensers are required at the ICU entry, exits, every bed space, and every workstation. 
- There should be separate medication preparation area.

- There should be separate areas for clean storage, soiled and waste storage and disposal.

- Adequate toilet facilities should be provided.

\section{Step 9: Organizational and Administrative Measures}

- Work with hospital administration for better patient-to-nurse ratio in the ICU.

- Policies for controlling traffic flow to and from the unit to reduce sources of contamination from visitors, staff, and equipment.

- Waste and sharp disposal policy.

- Education and training for ICU staff about prevention of nosocomial infections.

- ICU protocols for prevention of nosocomial infections.

- Audit and surveillance of infections and infection control practices.

- Infection control team (multidisciplinary approach).

- Antibiotic stewardship.

- Vaccination of health-care personnel.

\section{Antibiotic Stewardship}

Antibiotic stewardship has been defined as "coordinated interventions designed to improve and measure the appropriate use of antibiotic agents by promoting the selection of the optimal antibiotic drug regimen including dosing, duration of therapy, and route of administration".

Antibiotic stewardship ensures the right drug in right dose at the right time for the right duration for any infection, thereby eradicating infection with minimial side effects. It consists of prospective audit and feedback, education, antibiotic restriction, antibiotics de-escalation, guideline use, optimal dosing and duration, microbiologist, and computer aided clinical support. Properly executed, this is associated with reduced drug resistance, improved patient outcome, and optimized resource utilization. These policies have shown to improve antibiotic prescribing and drug resistance patterns without an increase in patient mortality or hospital length of stay.

\section{Suggested Reading}

Johnson I. Banks V; Antibiotic stewardship in critical care. BJA Educ. 2017;17(4):111-6.

Klompas M, Branson R, Eichenwald EC, Greene LR, Howell MD, Lee G, Magill SS, Maragakis LL, Priebe GP, Speck K, Yokoe DS, Berenholtz SM. Strategies to prevent ventilator-associated pneumonia in acute care hospitals: 2014 update. Infect Control Hosp Epidemiol. 2014;35(Suppl 2):S133-54. Describes Strategies for prevention of ventilator associated pneumonia.

Ling ML, Apisarnthanarak A, Jaggi N, et al. APSIC guide for prevention of Central Line Associated Bloodstream Infections (CLABSI). Antimicrob Resist Infect Control. 2016;5:16. https://doi. 
org/10.1186/s13756-016-0116-5. Guide for the prevention of central line associated blood stream infections.

Mehta Y, Gupta A, Todi S, Myatra SN, Samaddar DP, Patil V, Bhattacharya PK, Ramasubban S. Guidelines for prevention of hospital acquired infections. Indian J Crit Care Med. 2014;18:149-63. Describes Indian guidelines for prevention of hospital acquired infections.

Torres A, Niederman MS, Chastre J, Ewig S, Fernandez-Vandellos P, Hanberger H, Kollef M, Bassi GL, Luna CM, Martin-Loeches I, ArturPaiva J, Read RC, Rigau D, Timsit JF, Welte $\mathrm{T}$, Wunderink R. Summary of the international clinical guidelines for the management of hospital-acquired and ventilator-acquired pneumonia. ERJ Open Research. 2018;4(2):000282018. https://doi.org/10.1183/23120541.00028-2018. Guidelines for the management of hospital acquired and ventilator associated pneumonia.

Antoni Torres, Michael S. Niederman, Jean Chastre, Santiago Ewig, Patricia Fernandez-Vandellos, Hakan Hanberger, Marin Kollef, Gianluigi Li Bassi, Carlos M. Luna, Ignacio MartinLoeches, J. ArturPaiva, Robert C. Read, David Rigau, Jean François Timsit, Tobias Welte, Richard Wunderink. International ERS/ESICM/ESCMID/ALAT guidelines for the management of hospital-acquired pneumonia and ventilator-associated pneumonia. Eur Respir J 2017;3:1700582. https://doi.org/10.1183/13993003.00582-2017. Guidelines for management of ventilator associated pneumonia.

Kalil AC, Metersky ML, Klompas M, Muscedere J, Sweeney DA, Palmer LB, Napolitano LM, O'Grady NP, Bartlett JG, Carratalà J, El Solh AA, Ewig S, Fey PD, File TM, Restrepo MI, Roberts JA, Waterer GW, Cruse P, Knight SL, Brozek JL. Management of adults with hospital-acquired and ventilator-associated pneumonia: 2016 clinical practice guidelines by the Infectious Diseases Society of America and the American Thoracic Society. Clin Infect Dis. 2016;63(5):61-e111. https://doi.org/10.1093/cid/ciw353. Guidelines for the management of hospital acquired and ventilator associated pneumonia.

\section{Websites}

http://whqlibdoc.who.int/hq/2009/WHO_IER_PSP_2009.07_eng.pdf

WHO guidelines on hand hygiene in health care: a summary.

http://www.cdc.gov/hicpac/pdf/guidelines/bsi-guidelines-2011.pdf

Guidelines for the prevention of intravascular catheter-related infections.

http://www.cdc.gov/hicpac/pdf/CAUTI/CAUTIguideline2009final.pdf

Guidelines for prevention of catheter-associated urinary tract infections.

http://theific.org/wp-content/uploads/2016/04/18-UTI_2016.pdf

Prevention of CatheterAssociated Urinary Tract Infections.

http://www.cdc.gov/hicpac/pdf/isolation/Isolation2007.pdf

Guideline for isolation precautions: preventing transmission of infectious agents in healthcare settings.

https://www.cdc.gov/infectioncontrol/guidelines/bsi/recommendations.html

Updated guidelines forprevention of catheter related infections.

https://www.cdc.gov/infectioncontrol/guidelines/environmental/appendix/air.html\#tableb2

Ventilation requirements for areas affecting patient care in hospitals and outpatient facilities. 